\title{
BMJ Open Status and influencing factors of diabetes information acquisition among rural elderly with pre-diabetes in Yiyang City, China: a cross- sectional study
}

Tingting Zhu, Huilan $\mathrm{Xu}^{\odot}$

To cite: Zhu T, Xu H. Status and influencing factors of diabetes information acquisition among rural elderly with pre-diabetes in Yiyang City, China: a crosssectional study. BMJ Open 2019;9:e029938. doi:10.1136/ bmjopen-2019-029938

- Prepublication history for this paper is available online. To view these files, please visit the journal online (http://dx.doi. org/10.1136/bmjopen-2019029938).

Received 18 February 2019 Revised 16 June 2019 Accepted 28 June 2019

Check for updates

(C) Author(s) (or their employer(s)) 2019. Re-use permitted under CC BY-NC. No commercial re-use. See rights and permissions. Published by BMJ.

Department of Social Medicine and Health Management, Xiangya School of Public Health, Central South University, Changsha, Hunan, China

Correspondence to Professor Huilan Xu; XuHuilan6319@163.com

\section{ABSTRACT}

Objective This study was designed to investigate the status of diabetes information acquisition and its influencing factors among patients, 60 years and older, with pre-diabetes in rural China.

Design This is a cross-sectional study.

Setting The survey was conducted in 42 rural communities in Yiyang City, Hunan Province, China. Participants A total of 461 elderly with pre-diabetes participated in this study, and 434 of them completed the survey $(434 / 461,94.1 \%)$.

Outcome measures Information on sociodemographic and diabetic information-seeking behaviours were assessed through the structured questionnaires and described by percentages, mean $\pm S D$. The influencing factors of diabetes information acquisition were analysed using multiple linear regression analysis.

Results The average score of diabetes information acquisition in the subjects was $8.39 \pm 11.28$. The most popular and trusted source of diabetes information for the subjects was doctors. The level of information acquisition not only declined as the age progressed $(95 \% \mathrm{Cl}-3.754$ to -0.143 ) but also proved lowest among subjects with less than 1 year of education $(95 \% \mathrm{Cl} 0.756$ to 4.326$)$. In addition, the level of information acquisition among elderly individuals with pre-diabetes, but with no history of hyperglycaemia, was lower than among those with a history of hyperglycaemia (95\% Cl 3.398 to 11.945$)$. Conclusions These data indicated that the lack of ability to acquire diabetes information in elderly with pre-diabetes was common in rural China. Improving the ability of rural residents to access information should be incorporated into rural diabetes prevention efforts, especially for the elderly with pre-diabetes with low education and no history of hyperglycaemia.

Trial registration number ChiCTR-IOR-15007033; Preresults.

\section{INTRODUCTION}

'Pre-diabetes' refers to blood sugar level that is above the normal, but at the same time falls below the threshold level of diabetic blood sugar. ${ }^{1}$ Individuals with impaired fasting
Strengths and limitations of this study

- This is the first study to assess the behaviour of diabetes information acquisition among the elderly with pre-diabetes in the rural areas of China.

- This study provides useful information for the government to identify special population that should be concerned when improving the ability to acquire information about diabetes in rural pre-diabetes.

- This is a cross-sectional study conducted in Yiyang city; we cannot know the causal relationship between variables and speculate on the situation in urban areas.

- The blood glucose levels of the subjects were measured in the laboratory of the primary care centre rather than the reference laboratory, which may have more or less impact on the outcome of pre-diabetes screening, affecting the number of study populations.

- This study only investigated the internal factors influencing diabetes information acquisition of the subjects.

glucose (IFG) or impaired glucose tolerance (IGT) have been referred to as having pre-diabetes, indicating the relatively high risk for the future development of diabetes. ${ }^{2}$ According to the data released by the International Diabetes Federation (IDF) in 2017, $7.3 \%$ of adults (20-79 years) were people with IGT and $72.3 \%$ of these people lived in low-income and middle-income countries. ${ }^{2}$ In 2017 , there were about 114 million people with diabetes and 48.6 million people with pre-diabetes in China, among whom the proportion of the rural elderly aged 60 and above was relatively large. ${ }^{2}$ A research conducted in England has confirmed that $33 \%$ of people with IFG and $65 \%$ of people with IGT will develop into type 2 diabetes within 6 years. ${ }^{3}$ And around $5 \%-10 \%$ of people with pre-diabetes developed diabetes every year. ${ }^{3}$ So, 
without timely and effective prevention, the prevalence of type 2 diabetes will increase significantly in the rural areas of China. ${ }^{1}$

The prevention of diabetes transformation requires patients with pre-diabetes to have a strong self-management ability, and the premise for a better grasp of this ability is having the relevant diabetes information at hand. ${ }^{4}$ Studies have identified the central role of information seeking and acquisition in enabling a person to cope with both the initial diagnosis and the ongoing impacts of diabetes. ${ }^{5}$ Although diabetes-related information plays an important role in prevention of diabetes, blood glucose monitoring, dietary care and other aspects, many elderly with pre-diabetes are not fully aware of this information and lack the necessary self-management skills, resulting in the conversion of diabetes, even worse. ${ }^{6}$ In rural areas of China, primary healthcare is based on township hospitals and village clinics, providing services on public health and basic medical care with a focus on disease prevention and health promotion, which have been vigorously developed, but there are still a series of problems such as large urban-rural gap, narrow coverage of healthcare, inadequate construction of facilities and single access to health information. ${ }^{78}$

The purpose of this cross-sectional study was to explore the status of diabetes information acquisition and its influencing factors among elderly patients with pre-diabetes in rural areas of Yiyang City, China. This study was conceived as a first step towards understanding these issues that could provide useful information for relevant departments to find key populations that should receive more attention when improving the ability to acquire diabetes information.

\section{METHODS}

\section{Sampling}

This survey was conducted during the period April 2015 to July 2015 and included a representative sample of the rural elderly population based on the multistage cluster random sampling method. At the first stage, three counties were randomly selected from the seven counties in Yiyang City; at the second stage, four townships were then randomly chosen from each county; and at the third stage, three or four villages were randomly selected from each township (about $30-50$ villages in each township), a total of 42 villages were selected as survey sites.

Subjects aged 60 years and above were eligible for inclusion in this study if they met the diagnostic criteria of pre-diabetes, ${ }^{9}$ including IFG $(6.1 \mathrm{mmol} / \mathrm{L} \leq$ fasting blood glucose $<7.0 \mathrm{mmol} / \mathrm{L})$ or IGT $(7.8 \mathrm{mmol} / \mathrm{L} \leq 2$-hour postprandial blood glucose $<11.1 \mathrm{mmol} / \mathrm{L})$. Patients who could not complete the communication tasks of this study due to severe physical illness, hearing impairment or cognitive impairment (such as stroke, deafness, dementia, psychosis) were excluded.

There were 3197 elderly in the selected villages, excluding those who could not be included in the study because of relocation (603), serious illness (336) and refusal to participate (114), leaving 2144 aged as screening samples $(67.1 \%)$. A total of 461 individuals with pre-diabetes were screened out $(21.5 \%)$. For some reason, 21 of them have not been investigated, and 6 of the 440 individuals were excluded due to incomplete data. Finally, data from 434 subjects were included in statistical analysis $(94.1 \%)$.

\section{Data collection and measurements}

The main outcome measures in this study were participants' sociodemographic information and ability to obtain diabetes-related information. We measured fasting blood glucose values using oral glucose tolerance test (OGTT) and collected sociodemographic information using a structural questionnaire. The status of diabetes information acquisition was assessed using the Questionnaire of Health Literacy of Diabetes of the Public in China ${ }^{10}$ designed by the Chinese Centre for Health Education, which has a high reliability and validity with a Cronbach's $\alpha$ of 0.866 . Our trained interviewers visited the elderly subjects' home to carefully explain the purpose and benefits of this study and participants' rights for elderly and family members. Participants were then interviewed face to face after giving written informed consent. If the participants were illiterate or they could not understand what the interviewers said about this study, the consent form was signed by a family member. The elderly could decline to participate in the study without penalty and could drop out at any time during the investigation.

\section{Pre-diabetes screening}

We mainly tested the level of fasting blood glucose and 2-hour postprandial blood glucose. The participants were instructed to maintain their usual physical activity and diet for a minimum of 3 days before the OGTT. After overnight fasting of at least 10 hours, the fasting blood samples of the participants were collected in the morning, and then each participant was orally administered $75 \mathrm{~g}$ of anhydrous glucose powder dissolved in $300 \mathrm{~mL}$ of water within 5 min. ${ }^{11}$ The venous blood were taken from the forearm on fasting and 2 hours after oral glucose; the blood samples were stored at $-80^{\circ} \mathrm{C}$ and measured for blood glucose levels $(\mathrm{mmol} / \mathrm{L})$ in the biochemical laboratory of the primary care centre in each village within 1 hour. Blood glucose levels were measured by the standardised hexokinase enzymatic method using the same reagents to avoid assay variations.

\section{Sociodemographic information}

Age, gender, marital status, occupation, individual annual income (Chinese Yuan), education, family history of diabetes, and other chronic diseases and history of hyperglycaemia were obtained for each subject. Marital status was grouped into two categories: stable (married and cohabiting) and unstable (living alone). Individual annual income refers to the total income earned by the individual in a year, divided into two categories according 
to the per capita annual net income of farmers in China in 2015. ${ }^{12}$ Other chronic diseases mean chronic diseases other than diabetes, such as cardiovascular disease (hypertension, coronary heart disease and stroke), malignant tumours and chronic obstructive pulmonary disease (chronic bronchitis, emphysema). Education was assessed by asking the participants to select their highest level of education completed from the following choices: less than 1 year, 1-6 years, 6 years and above.

\section{Diabetes information acquisition}

Thirteen questions are included in the section of diabetes information acquisition; the main contents of this section are related to factors influencing the acquisition of diabetes information, the channels for getting information, levels of trust in information sources and utilisation of the internet.

Five of the 13 questions were thus posed to extract the information needed:

1. How much do you know about diabetes?

2. Does your knowledge about diabetes meet your requirements?

3. Do you find it easy to find diabetes-related information when it is required?

4. Do you understand diabetes-related information?

5. Are you able to distinguish between correct and incorrect information about diabetes?

Each item was rated on a four-point scale, ranging from 0 to 3. Those who demonstrated good information acquisition were given a score of 3 , those with general acquisition a score of 2 and those with poor acquisition a score of 1 . Those who had not tried to find any diabetes-related information were given a score of 0 . In this section of the questionnaire, total scores ranged from 0 to 15 . Subjects' scores reflected their status of diabetes information acquisition, and subjects with a score of 8 or higher were defined as having great ability to acquire information regarding diabetes.

\section{Statistical analysis}

The data were analysed using SPSS V.18.0. Descriptive analysis of the basic sociodemographic information on subjects was expressed in terms of percentages, mean \pm SD. The $\mathrm{t}$-test and $\mathrm{F}$ test were used to explore differences in diabetes-related information acquisition among individual with pre-diabetes with different characteristics. Multiple linear regression analysis was performed to identify the influencing factors for diabetes-related information obtained among the rural elderly with pre-diabetes. We identified diabetes information acquisition scores as dependent variables and selected potential influencing factors as independent variables (age, marital status, education, other chronic disease, family history of diabetes, history of hyperglycaemia) in the analysis after controlling confounding factors (gender, individual annual income, job). All reported $\mathrm{p}$ values were from two-sided tests and compared with a significance level of $5 \%$.
Table 1 The basic demographic information of the subjects

\begin{tabular}{|c|c|c|}
\hline & $\mathbf{N}$ & Mean \pm SD $/ \%$ \\
\hline Age & 434 & $69.41 \pm 6.45$ \\
\hline \multicolumn{3}{|l|}{ Gender } \\
\hline Male & 180 & 41.47 \\
\hline Female & 254 & 58.53 \\
\hline \multicolumn{3}{|l|}{ Marital status } \\
\hline Stable & 313 & 72.12 \\
\hline Unstable & 121 & 27.88 \\
\hline \multicolumn{3}{|l|}{ Education } \\
\hline Less than 1 year & 81 & 18.66 \\
\hline 1-6years & 272 & 62.68 \\
\hline 6 years and above & 81 & 18.66 \\
\hline \multicolumn{3}{|c|}{ Individual annual income ( $¥)$} \\
\hline$\leq 2800$ & 90 & 20.73 \\
\hline$>2800$ & 344 & 79.27 \\
\hline \multicolumn{3}{|l|}{ Job } \\
\hline Farmer & 198 & 45.62 \\
\hline Worker & 100 & 23.04 \\
\hline Retired & 72 & 16.59 \\
\hline Other & 64 & 14.75 \\
\hline
\end{tabular}

Other chronic disease

\begin{tabular}{crr} 
Yes & 176 & 40.55 \\
\hline No & 258 & 59.45 \\
\hline Family history of diabetes & & \\
Yes & 36 & 8.29 \\
No & 398 & 91.71 \\
\hline
\end{tabular}

History of hyperglycaemia

\begin{tabular}{lrr} 
Yes & 28 & 6.45 \\
No & 406 & 93.55 \\
\hline
\end{tabular}

\section{Patient and public involvement}

Patients were not involved in developing the hypothesis, the aims or the research questions, nor were they involved in the design, interpretation of results or writing of manuscripts. There are no plans to disseminate the results of the research to study participants. Patients' involvement was related to the sole period of data collection.

\section{RESULTS}

The sociodemographic characteristics of the subjects are displayed in table 1 . The subjects' average age was $69.41 \pm 6.45$ years, of whom $45.62 \%$ were farmers, and $72.12 \%$ were married with stable marital status (not living alone). Most of the subjects had an education level of 1-6 years $(62.68 \%)$ and had a personal annual income of $¥ 2800$ or more $(79.27 \%)$. Only a small part of the sample had a family history of diabetes $(8.29 \%)$ and a history of high blood sugar $(6.45 \%)$. 
Table 2 The sources for subjects to acquire diabetes information

\begin{tabular}{ll}
\hline $\begin{array}{l}\text { The source of diabetes information } \\
\text { (multiple choice) }\end{array}$ & $\%$ \\
\hline Doctors & 82.82 \\
\hline Family members & 10.36 \\
\hline Newspapers or books & 2.30 \\
\hline Television or radio & 6.91 \\
\hline Network or mobile phone text messages & 0.46 \\
\hline
\end{tabular}

The status of the subjects' diabetes information acquisition is shown in tables 2 and 3 .

Most of the subjects were concerned about diabetes information because of the fact that their relatives, friends or colleagues have diabetes $(40.10 \%)$; some subjects were worried about getting diabetes in the future $(36.71 \%)$. And doctors were the most important and trusted source of diabetes information for the subjects $(82.82 \%)$. Some subjects obtained information through family members $(10.36 \%)$, newspapers or books $(2.30 \%)$, television or radio $(6.91 \%)$. Almost no subject relied on web or mobile phone text messages $(0.46 \%)$. The average score of diabetes information acquisition was $8.39 \pm 11.28$, the lowest score was 0 and the highest score was 12 . The differences in diabetes information acquisition scores among different characteristics were examined. Results demonstrated that age $(\mathrm{F}=3.747, \mathrm{p}=0.024)$, education level $(\mathrm{F}=6.492, \mathrm{p}=0.002)$ and history of hyperglycaemia $(t=-3.967, \mathrm{p}=0.000)$ were associated with diabetes information acquisition scores.

The results of the multiple linear regression analysis of the influencing factors for diabetes-related information acquisition are presented in table 4 . We found that the elderly subjects with pre-diabetes who were older $(B=-1.948, \mathrm{p}=0.034)$, had a lower educational level $(B=2.541, \mathrm{p}=0.005)$ and did not have a history of hyperglycaemia $(B=7.671, \mathrm{p}=0.000)$ were more likely to have poorer ability to access information on diabetes.

\begin{tabular}{lrrrr}
$\begin{array}{l}\text { Table 4 } \\
\text { subjects }\end{array}$ & The diabetes information acquisition scores of the \\
\hline Model 1 & $\boldsymbol{B}$ & $\boldsymbol{t}$ & $\mathbf{P}$ value & $\mathbf{9 5 \%} \mathbf{~ C l}$ \\
\hline Age & -0.102 & -2.095 & 0.037 & -3.698 to -0.118 \\
$\begin{array}{l}\text { Education } \\
\text { History of } \\
\text { hyperglycaemia }\end{array}$ & 0.139 & 2.894 & 0.004 & 0.822 to 4.302 \\
\hline Model 2 & $\mathbf{B}$ & $\mathbf{t}$ & $\mathbf{P}$ value & $\mathbf{9 5 \%} \mathbf{C l}$ \\
\hline Age & -0.104 & -2.121 & 0.034 & -3.754 to -0.143 \\
\hline $\begin{array}{l}\text { Education } \\
\text { History of }\end{array}$ & 0.138 & 2.798 & 0.005 & 0.756 to 4.326 \\
hyperglycaemia & 0.167 & 3.529 & $<0.01$ & 3.398 to 11.945 \\
\hline
\end{tabular}

Model 1: the crude result before controlling for confounders.

Model 2: the result after controlling for confounders.
Table 3 The diabetes information acquisition scores of the subjects

\begin{tabular}{llll}
\hline & Mean \pm SD & t/F & P value \\
\hline Age & & 3.747 & 0.024 \\
\hline $60 \sim$ & $9.62 \pm 12.56$ & & \\
$70 \sim$ & $7.23 \pm 9.59$ & & \\
80 & $4.58 \pm 6.74$ & & \\
Gender & & 0.219 & 0.827 \\
$\quad$ Male & $8.53 \pm 12.57$ & & \\
Female & $8.29 \pm 10.29$ & & \\
Marital status & & 1.281 & 0.201 \\
\hline Stable & & & \\
Unstable & $7.82 \pm 11.43$ & & \\
\hline Education & & & \\
\hline Less than 1 year & $5.12 \pm 7.37$ & & \\
\hline 1-6years & $8.56 \pm 11.09$ & & \\
\hline 6years and above & $11.42 \pm 14.10$ & & \\
\hline
\end{tabular}

$\begin{array}{lll}\text { Individual annual } & -0.470 & 0.638 \\ \text { income }(¥) & & \end{array}$

\begin{tabular}{|lcll|}
\hline$\leq 2800$ & $7.89 \pm 10.19$ & & \\
\hline 2800 & $8.52 \pm 11.55$ & & \\
\hline Job & & 0.421 & 0.738 \\
\hline Farmer & $8.36 \pm 11.44$ & & \\
\hline Worker & $7.90 \pm 12.62$ & & \\
\hline Retired & $7.92 \pm 9.37$ & & \\
\hline Other & $9.77 \pm 10.63$ & & \\
\hline $\begin{array}{l}\text { Other chronic } \\
\text { disease }\end{array}$ & & -1.486 & 0.138 \\
\hline Yes & $9.05 \pm 10.65$ & & \\
\hline No & $7.41 \pm 12.11$ & & \\
$\begin{array}{l}\text { Family history of } \\
\text { diabetes }\end{array}$ & & -1.593 & 0.112 \\
\hline Yes & $12.86 \pm 6.80$ & & \\
\hline No & $8.13 \pm 11.32$ & & \\
\hline $\begin{array}{l}\text { History of } \\
\text { hyperglycaemia }\end{array}$ & & -3.967 & 0.000 \\
\hline Yes & $16.43 \pm 11.93$ & & \\
\hline No & $7.83 \pm 11.03$ & & \\
\hline
\end{tabular}

\section{DISCUSSION}

Self-management knowledge and skills in patients with pre-diabetes depend on high quality health information, which is important for glycaemic control. ${ }^{13}$ Although information alone is not enough to change behaviour and improve outcomes, it is impossible to achieve desirable behavioural changes without appropriate access to diabetes information and services, resulting in poor selfcare and ultimately poor clinical outcomes. ${ }^{14}$ We used a questionnaire to measure diabetes-related information acquisition among patients with pre-diabetes aged 60 and over in rural areas of Yiyang City, Hunan Province. 
The randomisation of sampling and the consistency of survey methods were used to control the selection bias and information bias which are common in cross-sectional studies to reduce errors in this study. The average score of diabetes-related information acquisition was $8.39 \pm 11.28$, the lowest score was 0 and the highest score was 12 , suggesting that the ability of elderly rural individuals with pre-diabetes to acquire information was limited to a lower level.

The main and most trusted source of diabetes information for the elderly with pre-diabetes in rural areas was doctors, which was similar to the previous studies. ${ }^{15-18}$ In addition, some previous research has found that family members, ${ }^{19}$ neighbours, ${ }^{20}$ magazines,${ }^{21}$ television $^{21}$ and the internet ${ }^{22} 23$ were the sources of health or disease information as much for the elderly as for other groups. As technology advances, it is becoming more and more common for individuals to use the internet or mobile phones to search for relevant health information. Internet transmission of diabetes information in China is mainly based on the websites related to diabetes or health. ${ }^{24}$ From 2009 to 2015, the internet penetration rate in rural China was relatively stable, with the number of elderly internet users over 60 years old rising from below $1 \%$ to $4 \% .{ }^{25}$ Internet and mobile phones play a significant role in obtaining information, improving livelihoods and accelerating development in rural areas. ${ }^{26}$ However, access to internet or mobile phone information depends, to a large extent, on individual education background, age, household income, infrastructure and other factors among rural residents, ${ }^{27}$ and disease information resources are more scarce in rural areas. Thus, subjects in this study rarely used internet or mobile phones to obtain information about diabetes. Research has confirmed that the doctors remain the most popular and trusted source of health information available to the elderly with limited knowledge of diabetes. ${ }^{28}{ }^{29}$ In the areas we surveyed, each village has a primary care centre with one to two village doctors. Due to the lack of professional healthcare personnel, public health work such as health information dissemination and health education cannot be implemented, and rural residents have little chance to understand their health status and gain knowledge about diabetes prevention. Therefore, it follows that there is still a need for the departments of public health to step up their efforts in equipping primary healthcare workers in rural China with knowledge and skills related to diabetes.

Numerous studies have found that factors such as technology, ${ }^{30}$ community environment, ${ }^{31}$ family history of diabetes,${ }^{32}$ health literacy, ${ }^{33}$ cultural beliefs ${ }^{34}$ and anxiety ${ }^{35}$ have an impact on the ability of patients with diabetes to obtain diabetes-related information. Our study found that age is an influencing factor of diabetes-related information acquisition among the rural elderly with pre-diabetes, probably because the aged progressively weaken the ability to access information as they grow older. A previous research on health education in rural America also found that age has a negative effect on the acquisition of information. ${ }^{36}$ In the case of people getting older, it is necessary not only to pay more attention to the elderly but also to strengthen public health interventions for the younger generation. We also found that the accessibility of diabetes-related information for the elderly with pre-diabetes was influenced by educational level and history of hyperglycaemia. People with higher educational levels were more aware of health management and more motivated to obtain information. ${ }^{37}$ This result is similar to the 2013 study of elderly type 2 diabetes care in Vietnam. ${ }^{37}$ Old people with a history of hyperglycaemia had more opportunities to contact medical staffs to better understand diabetes-related information. Similar findings have not been demonstrated in other studies.

This is the first study to assess the behaviour of the diabetes information acquisition among the elderly with pre-diabetes in the rural areas of China and provides useful information for the government to identify special population that should be concerned when improving the ability to acquire information about diabetes in rural pre-diabetes. Several limitations in this study should be considered when interpreting these findings. First, this is a cross-sectional study conducted in the rural area of Yiyang City, Hunan Province, so we cannot infer any causal relationship between the study variables and speculate on the situation in urban areas based on the results of the study. Second, all information was self-reported, which could lead to reporting bias, meaning that sometimes the elderly were unwilling to honestly answer the questions asked by the investigator for some reason, which may cause errors in the results. Third, we used the laboratory of the primary care centre instead of the reference laboratory to measure subjects' blood glucose levels, which may cause a little assay variation, affecting the number of study populations. Fourth, this study only investigated the internal factors that affect diabetes information acquisition of the subjects and did not assess and analyse the external factors such as healthcare provider, diabetes management level and healthcare facilities.

\section{CONCLUSIONS}

We concluded that the ability of the elderly to obtain diabetes information in rural areas was not optimistic, and doctors were their primary and most trusted source of diabetes information. Age, educational level and history of hyperglycaemia were the factors affecting the information acquisition behaviour of the subjects. It is clear that future studies are required to access the external factors affecting information acquisition among the elderly with pre-diabetes and other populations in rural China, and intervention studies on diabetes information in rural areas are equally needed. 
Contributors All the authors contributed to the study design and funding application. $\mathrm{HX}$ and $\mathrm{TZ}$ wrote, checked and revised the manuscript.

Funding This work was supported by the Central South University Teacher Research Fund Project (2013JSJJ034).

Competing interests None declared.

Patient consent for publication Not required.

Ethics approval This study was conducted in strict accordance with the Declaration of Helsinki. Approval was obtained from the Medical Ethics Committee of the Institute of Clinical Pharmacology, Central South University (No. CTXY150002-7) and the Institutional Review Board of the Chinese Clinical Trial Registry (No. ChiCTR-IOR-15007033). Prior to the investigation, through the oral explanationof interviewers, subjects understood the purpose of this study and voluntarily signed informed consent.

Provenance and peer review Not commissioned; externally peer reviewed. Data sharing statement Data are available on reasonable request.

Open access This is an open access article distributed in accordance with the Creative Commons Attribution Non Commercial (CC BY-NC 4.0) license, which permits others to distribute, remix, adapt, build upon this work non-commercially, and license their derivative works on different terms, provided the original work is properly cited, appropriate credit is given, any changes made indicated, and the use is non-commercial. See: http://creativecommons.org/licenses/by-nc/4.0/.

\section{REFERENCES}

1. Shaw JE, Sicree RA, Zimmet PZ. Global estimates of the prevalence of diabetes for 2010 and 2030. Diabetes Res Clin Pract 2010;87:4-14.

2. International Diabetes Federation. IDF Diabetes Atlas. 7th ed. Brussels, Belgium: International Diabetes Federation, 2017.

3. Tabák AG, Herder C, Rathmann W, et al. Prediabetes: a high-risk state for diabetes development. Lancet 2012;379:2279-90.

4. Wilson V. Patient use of the internet for diabetes information. Nurs Times 2013;109:18-20.

5. Kalantzi S, Kostagiolas P, Kechagias G, et al. Information seeking behavior of patients with diabetes mellitus: a cross-sectional study in an outpatient clinic of a university-affiliated hospital in Athens, Greece. BMC Res Notes 2015;8:48.

6. Milewski J, Chen Y. Barriers of obtaining health information among diabetes patients. Stud Health Technol Inform 2010;160:18-22.

7. Feng Z. Chinese health care in rural areas. BMJ 2010;341:c5254.

8. Wang T, Zeng R. Addressing inequalities in China's health service. Lancet 2015;386:1441.

9. American Diabetes Association. 2. Classification and Diagnosis of Diabetes. Diabetes Care 2017;40:S11-S24.

10. Li L, Li Y, Nie X, Nia X, et al. [An analysis of health literacy about diabetes prevention and control and its influencing factors among the residents in six provinces in China]. Zhonghua Yu Fang Yi Xue Za Zhi 2014:48:561-5.

11. American Diabetes Association. (2) Classification and diagnosis of diabetes. Diabetes Care 2015;38 Suppl:S8-S16.

12. China's poverty line standard in 2015: the annual per capita net income of farmers is 2.800 yuan 2015 http://gold.cngold.com.cn/ 20151216d1762n59506450.html.

13. Alam R, Speed S, Beaver K. A scoping review on the experiences and preferences in accessing diabetes-related healthcare information and services by British Bangladeshis. Health Soc Care Community 2012;20:155-71.

14. Knight KM, Dornan T, Bundy C. The diabetes educator: trying hard, but must concentrate more on behaviour. Diabet Med 2006;23:485-501.

15. Hang XG, Yh L, Li L, et al. Survey the current state about information acquisition and internet utilization of diabetic patients in six provinces of China. Chinese Journal of Health Education 2014:20-2.
16. De Silva AP, De Silva SHP, Haniffa R, et al. A survey on socioeconomic determinants of diabetes mellitus management in a lower middle income setting. Int $J$ Equity Health 2016;15:15.

17. Baig AA, Locklin CA, Campbell A, et al. Community health center access to resources for their patients with diabetes. J Immigr Minor Health 2014;16:553-8.

18. Jafari J, Karimi Moonaghi $\mathrm{H}$, Zary N, et al. Exploring educational needs and design aspects of internet-enabled patient education for persons with diabetes: a qualitative interview study. BMJ Open 2016;6:6.

19. Gele AA, Torheim LE, Pettersen KS, et al. Beyond culture and language: access to diabetes preventive health services among somali women in Norway. J Diabetes Res 2015;2015:1-9.

20. Gariepy G, Smith KJ, Schmitz N. Diabetes distress and neighborhood characteristics in people with type 2 diabetes. $J$ Psychosom Res 2013;75:147-52.

21. Cleveland AD, Philbrick J, Pan XD, et al. Quality health information on the internet: developing a diabetes pathfinder for the chinese population. J Consum Health Internet 2009;13:313-33.

22. McKay HG, Feil EG, Glasgow RE, et al. Feasibility and use of an Internet support service for diabetes self-management. Diabetes Educ 1998;24:174-9.

23. Gerber BS, Solomon MC, Shaffer TL, et al. Evaluation of an internet diabetes self-management training program for adolescents and young adults. Diabetes Technol Ther 2007;9:60-7.

24. Wei X, Wu H, Cui S, et al. Intelligent Internet-based information system optimises diabetes mellitus management in communities. Health Inf Manag 2018;47:70-6.

25. Lh Y. The characteristics of rural netizens in China. Western Radio and Television 2018:22-6.

26. Gb Q, Wang HM, Zuo T. Farmers' access to internet information: Pathways, interests and cost. International Federation for Information Processing 2008;259:1155-+.

27. Lin CW, Abdul SS, Clinciu DL, et al. Empowering village doctors and enhancing rural healthcare using cloud computing in a rural area of mainland China. Comput Methods Programs Biomed 2014;113:585-92.

28. Ledikwe JH, Miersch JL, Smiciklas-Wright H. Accuracy and completeness of diabetes self-care information detected searching the internet. J Nutr Elder 2002;22:1-13.

29. Sinclair AJ, Armes DG, Randhawa G, et al. Caring for older adults with diabetes mellitus: characteristics of carers and their prime roles and responsibilities. Diabet Med 2010;27:1055-9.

30. Adaji A, Schattner P, Jones K. The use of information technology to enhance diabetes management in primary care: a literature review. Inform Prim Care 2008;16:229-37.

31. Pollard SL, Zachary DA, Wingert K, et al. Family and community influences on diabetes-related dietary change in a low-income urban neighborhood. Diabetes Educ 2014;40:462-9.

32. Cántaro K, Jara JA, Taboada M, et al. Association between information sources and level of knowledge about diabetes in patients with type 2 diabetes. Endocrinol Nutr 2016;63:202-11.

33. Yom-Tov E, Marino B, Pai J, et al. The effect of limited health literacy on how internet users learn about diabetes. J Health Commun 2016;21:1107-14.

34. Grace C, Begum R, Subhani S, et al. Prevention of type 2 diabetes in British Bangladeshis: qualitative study of community, religious, and professional perspectives. BMJ 2008;337:a1931.

35. Merrell J, Kinsella F, Murphy F, et al. Support needs of carers of dependent adults from a Bangladeshi community. J Adv Nurs 2005;51:549-57.

36. Mcllhenny CV, Guzic BL, Knee DR, et al. Using technology to deliver healthcare education to rural patients. Rural Remote Health 2011;11:11.

37. Carolan-Olah MC, Cassar A, Quiazon R, et al. Diabetes care and service access among elderly Vietnamese with type 2 diabetes. BMC Health Serv Res 2013;13:13. 